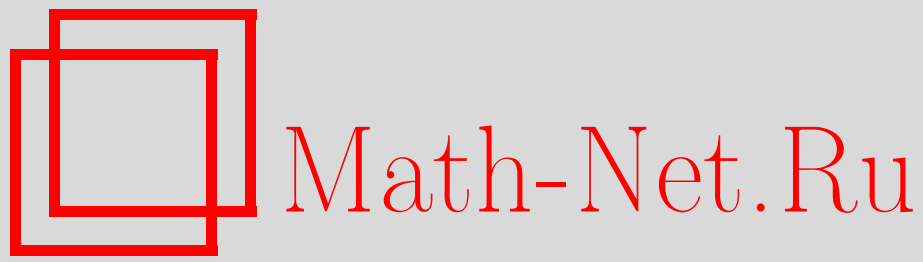

Б. Саймон, Мероморфные функции Йоста и асимптотические разложения для параметров Якоби, Функи. анализ и его прил., 2007, том 41, выпуск $2,78-92$

DOI: https://doi.org/10.4213/faa2862

Использование Общероссийского математического портала MathNet.Ru подразумевает, что вы прочитали и согласны с пользовательским соглашением

http://www . mathnet.ru/rus/agreement

Параметры загрузки:

IP : 34.239 .49 .27

26 апреля 2023 г., 13:13:54

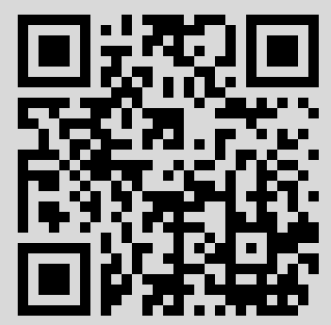




\title{
Мероморфные функции Йоста и асимптотические разложения для параметров Якоби*
}

\author{
(c) 2007. Б. САЙмон
}

Посвящается памяти М. Г. Крейна

\section{$\S 1$. Введение}

В данной работе мы рассматриваем полубесконечные матрицы Якоби

$$
J=\left(\begin{array}{cccc}
b_{1} & a_{1} & 0 & \ldots \\
a_{1} & b_{2} & a_{2} & \ldots \\
0 & a_{2} & b_{3} & \ldots \\
\vdots & \vdots & \vdots & \ddots
\end{array}\right)
$$

с экспоненциально убывающими параметрами Якоби (последнее означает, что $\left.\lim \sup _{n \rightarrow \infty}\left(\left|b_{n}\right|+\left|a_{n}-1\right|\right)^{1 / n}<1\right)$. Как обсуждалось в первых двух статьях [2], [3] этой серии (и было хорошо известно ранее), такой матрице $J$ можно сопоставить функцию Йоста $u$, определенную и аналитическую в окрестности замыкания $\overline{\mathbb{D}}$ круга $\mathbb{D}=\{z \in \mathbb{C}:|z|<1\}$.

Как обычно, $J$ описывает рекуррентные соотношения для ортогональных многочленов на вещественной прямой (ОМВП): существует такая вероятностная мера $\gamma$ на $\mathbb{R}$, что ортогональные многочлены $p_{n}(x)$ (см. [14], [10], [11]), построенные по этой мере, удовлетворяют уравнениям

$$
x p_{n}(x)=a_{n+1} p_{n+1}(x)+b_{n+1} p_{n}(x)+a_{n} p_{n-1}(x) .
$$

Мера $\gamma$ является спектральной мерой матрицы $J$, ассоциированной с вектором $(100 \ldots)^{\top}$, а $a_{n}$ и $b_{n}$ могут быть выражены через моменты этой меры при помощи процедуры Грама-Шмидта.

Функция Йоста $u$ определяется мерой $\gamma$ в силу следующих трех фактов:

(i) $u(z)=0, z \in \mathbb{D}$, тогда и только тогда, когда $z+z^{-1}-$ собственное число матрицы $J$;

(ii) носитель меры $d \gamma_{\mathrm{s}}$ (сингулярной части меры $d \gamma$ ) - конечное множество собственных чисел, лежащих в $\mathbb{R} \backslash[-2,2]$, и

$$
d \gamma \uparrow[-2,2]=f(x) d x \text {, }
$$

где

для всех $\theta \in[0,2 \pi)$;

$$
f(2 \cos \theta)=\frac{1}{\pi}\left[\frac{|\sin \theta|}{\left|u\left(e^{i \theta}\right)\right|^{2}}\right]
$$

(iii) $u(0)>0$.

*При частичной поддержке гранта NSF DMS-0140592 и гранта No. 2002068 Совместного американо-израильского научного фонда (BSF), Иерусалим, Израиль. 
Эти факты позволяют определить функцию $u$ (исходя из меры $\gamma$ ) при помощи стандартной теории «хороших» аналитических функций в $\mathbb{D}$. Напротив, функция $u$ во многих случаях не определяет меру $\gamma$. В силу соотношений (1.3), (1.4) она определяет абсолютно непрерывную составляющую этой меры и положение точечных нагрузок, но не веса. Мы предпочитаем нормализовать веса при помощи функции

$$
M(z)=-\int \frac{d \gamma(x)}{x-\left(z+z^{-1}\right)},
$$

рассматривая ее вычеты в тех точках, где $u(z)=0$. Исходно функция $M$ определена равенством (1.5) только для $z \in \mathbb{D}$. Это обычная $m$-функция, перенесенная в $\mathbb{D}$ при помощи стандартного отображения.

Так или иначе, функция $u$ и веса составляют спектральные данные, и наша задача - установить эквивалентность между этими спектральными данными, с одной стороны, и коэффициентами в рекуррентных соотношениях - с другой.

Для того чтобы сформулировать основную теорему, нам необходимо

Определение. Будем говорить, что последовательность $\left(x_{0}, \ldots, x_{n}, \ldots\right)$ комплексных чисел обладает асимптотическим разложением вплоть до $R>1$, ecли существуют такие числа $\mu_{1}, \ldots, \mu_{K(R)} \in\{z: 1<|z|<R\}$ и многочлены $p_{1}, \ldots$, $p_{K(R)}$, что

$$
\limsup _{n \rightarrow \infty}\left|x_{n}-\sum_{j=1}^{K(R)} p_{j}(n) \mu_{j}^{-n}\right|^{1 / n} \leqslant R^{-1} .
$$

Будем говорить, что $\left(x_{0}, \ldots\right)$ обладает полным асимптотическим разложением, если такие числа и многочлены существуют для каждого $R>1$.

Легко видеть, что числа $x_{n}$ однозначно определяют $p_{j}$ и $\mu_{j}$ и что справедлива

Теорема 1.1. Последовательность $\left\{x_{n}\right\}_{n=0}^{\infty}$ обладает асимптотическим разложением вплоть до $R$ в том и только том случае, когда функиия

$$
f(z) \equiv \sum_{n=0}^{\infty} x_{n} z^{n}
$$

мероморбна в $\{z:|z|<R\}$, имеет в этой области лишь конечное число полюсов и не имеет особенностей в окрестности круга $\overline{\mathbb{D}}$. Последовательность $\left\{x_{n}\right\}_{n=0}^{\infty}$ обладает полньм асимптотическим разложением в том и только том случае, когда функиия $f$ мероморфна в $\mathbb{C}$.

Действительно, полюсами функции $f$ являются $\mu_{j}$, а их порядками - увеличенные на 1 степени многочленов $p_{j}$.

Мы говорим, что параметры Якоби обладают асимптотическим разложением вплоть до $R$, если последовательность

$$
\left(1,-b_{1}, 1-a_{1}^{2},-b_{2}, 1-a_{2}^{2}, \ldots\right)
$$

обладает асимптотическим разложением вплоть до $R$. Таким образом, соответствующая функция $f-$ это

$$
B(z)=1-\sum_{n=0}^{\infty}\left[b_{n+1} z^{2 n+1}+\left(a_{n+1}^{2}-1\right) z^{2 n+2}\right] .
$$


Эта функция естественным образом появится ниже, а сейчас мы отметим следующую интерпретацию: если $J_{0}-$ матрица Якоби с $a_{n} \equiv 1, b_{n} \equiv 0$, а $\delta J=J-J_{0}$, то (см. лемму 6.2 в [2])

$\operatorname{Tr}\left(\delta J\left(J_{0}-\left(z+z^{-1}\right)\right)^{-1}\right)=-\left(z^{-1}-z\right)^{-1}\left\{\sum_{n=1}^{\infty} b_{n}\left(1-z^{2 n}\right)+2 \sum_{n=1}^{\infty}\left(a_{n}-1\right)\left(z-z^{2 n+1}\right)\right\}$.

Кроме того (см. теорему 2.16 в [9]),

$$
u(z)=\left(\prod_{j=1}^{\infty} a_{j}\right)^{-1} \operatorname{det}\left(1+\delta J\left(J_{0}-\left(z+z^{-1}\right)\right)^{-1}\right) .
$$

Принимая во внимание, что $a_{n}^{2}-1=2\left(a_{n}-1\right)+O\left(\left(a_{n}-1\right)^{2}\right)$ и $\operatorname{det}(1+A)=$ $1+\operatorname{Tr}(A)+O\left(\|A\|_{1}^{2}\right)$, мы видим, что если $\delta J-$ ядерный оператор, то

$$
-\left(z^{-1}-z\right)\left(\prod_{j=1}^{\infty} a_{j}\right) u(z)=c(z)+z B(z)+O\left(\|\delta J\|_{1}^{2}\right)
$$

для некоторой аффинной функции $c(z)$. То есть $B(z)$ является некоторой (борновской) аппроксимацией первого порядка функции $u$.

В каком-то смысле основным результатом данной работы является

Теорема 1.2. Параметры Якоби обладают полным асимптотическим разложением в том и только том случае, когда и - мероморфнал в $\mathbb{C}$ функиия. Другими словами, В(z) мероморфна в $\mathbb{C}$ тогда и только тогда, когда мероморфной является и(z).

Конечно, хотелось бы установить связь между полюсами функций $u$ и $B$. Отчасти для этого, отчасти поскольку мы все равно будем использовать их в доказательствах (§3), имеет смысл привести наши недавние результаты из [12] об аналогичной задаче для ортогональных многочленов на единичной окружности (OMEO). Хорошо известно (подробнее см. в [10] и [11]), что с каждой нетривиальной вероятностной мерой $\mu$ на $\partial \mathbb{D}$ связана последовательность коэффициентов Верблунского, определяемых формулами

$$
\Phi_{n+1}(z)=z \Phi_{n}(z)-\bar{\alpha}_{n} \Phi_{n}^{*}(z),
$$

где через $\Phi_{n}$ обозначены нормированные ортогональные (относительно $\mu$ ) многочлены и

$$
\Phi_{n}^{*}(z)=z^{n} \overline{\Phi_{n}(1 / \bar{z})} .
$$

Вместо функции $B$ в [12] используется функция

$$
S(z)=1-\sum_{j=1}^{\infty} \alpha_{j-1} z^{j},
$$

а вместо $u$ - функция Сегё

$$
D(z)=\exp \left(\int \frac{e^{i \theta}+z}{e^{i \theta}-z} \log (w(\theta)) \frac{d \theta}{4 \pi}\right)
$$


где $d \mu=w(\theta) \frac{d \theta}{2 \pi}+d \mu_{\mathrm{s}}$. Положим

$$
r(z)=\frac{D^{-1}(z)}{\overline{D^{-1}(1 / \bar{z})}} .
$$

Основными в [12] являются следующие теоремы 1.3 и 1.4 .

Теорема 1.3 [4]. Если $\lim \sup \left|\alpha_{n}\right|^{1 / n}=R^{-1}<1$, mо $r(z)-S(z)$ аналитична в $\left\{z: 1-\delta<|z|<R^{3}\right\}$ для некоторого $\delta>0$.

Замечания. 1. Этот результат принадлежит Дейфту и Остенссону [4], однако в [12] содержится его новое доказательство. Ранее в [10] был доказан более слабый результат, с $R^{2}$ вместо $R^{3}$.

2. Суть дела состоит в том, что обе функции $r$ и $S$ имеют особенности на окружности $|z|=R$. Теорема утверждает, что они сокращаются, равно как и все особенности в кольце $\left\{z: R<|z|<R^{3}\right\}$.

3. В [12] приведены явные примеры, в которых $r(z)-S(z)$ имеет особенности на $\left\{z:|z|=R^{3}\right\}$, и показано, что так же обстоит дело и в общем случае. Таким образом, $R^{3}$ - наилучшая возможная граница.

Для данного дискретного (с возможной предельной точкой лишь в $\infty$ ) множества $\Omega \subset\{z:|z|>1\}$ мы полагаем

$$
\begin{aligned}
\mathbb{G}^{2 j-1}(\Omega) & =\left\{\mu_{1} \ldots \mu_{j} \bar{\mu}_{j+1} \ldots \bar{\mu}_{2 j-1}: \mu_{k} \in \Omega\right\}, \\
\mathbb{G}(\Omega) & =\bigcup_{j=1}^{\infty} \mathbb{G}^{2 j-1}(\Omega) .
\end{aligned}
$$

Теорема 1.4 [12]. Функиия $S$ является иелой в том и только том случае, когда целой является $D^{-1}$. Если $T$ - множество полюсов функции $S(z), a$ $P$ - множество полюсов функиии $D^{-1}(z)$, то

$$
T \subset \mathbb{G}(P), \quad P \subset \mathbb{G}(T) .
$$

В 22 мы доказываем следующий сходный с теоремой 1.3 результат:

Теорема 1.5. Предположсим, что

$$
\limsup _{n \rightarrow \infty}\left(\left|a_{n}^{2}-1\right|+\left|b_{n}\right|\right)^{1 / 2 n}=R^{-1}<1 .
$$

Тогда функиия

$$
\left(1-z^{2}\right) u(z)+z^{2} \overline{u(1 / \bar{z})} B(z)
$$

аналитична в $\left\{z: R^{-1}<|z|<R^{2}\right\}$.

Замечания. 1. В работе [3] содержатся необходимые и достаточные условия на $u$ и веса, при которых выполняется соотношение (1.21). Если у $J$ нет собственных значений вне $[-2,2]$, то необходимым и достаточным условием является аналитичность функции $u$ в $\{z:|z|<R\}$.

2. Функция $u$ вещественна на $\mathbb{R}$; поэтому $\overline{u(\bar{z})}=u(z)$ и, следовательно, функцию (1.22) можно записать как $\left(1-z^{2}\right) u(z)+u(1 / z) B(z)$; мы используем выражение (1.22), чтобы подчеркнуть аналогию со случаем OMEО.

3. Конечно, у $B$ есть особенности на окружности $\{z:|z|=R\}$. Таким образом, теорема 1.5 утверждает, что они сокращаются либо с нулями функции $\overline{u(1 / \bar{z})}$, либо с особенностями функции $u$. Так как $\overline{u(1 / \bar{z})}$ может иметь нули в

4 Функциональный анализ и его приложения, т. 41, вып. 2 
$|z|>1$ (в отличие от $\overline{D(\bar{z})^{-1}}$ ), ситуация здесь несколько иная, чем в случае OMEО. Мы обсуждаем это в $§ 2$.

4. Как показано в $\S 3$, функция (1.22) часто имеет особенности на окружности $\left\{z:|z|=R^{2}\right\}$, так что $R^{2}$ нельзя увеличить до $R^{3}$, как в случае ОMЕО. Причины этого различия станут ясны в $\S 3$.

Для того чтобы сформулировать аналог теоремы 1.4, нам нужно большее множество, чем $\mathbb{G}$. В нашем случае $u$ и $B$ вещественны на $\mathbb{R}$, поэтому их полюсы симметричны относительно $\mathbb{R}$. Имея это в виду, предположим, что $\Omega \subset\{z$ : $|z|>1\}$ имеет предельную точку лишь в бесконечности и

$$
\bar{\Omega}=\Omega \text {. }
$$

Для каждого $m$ мы полагаем

$$
\mathbb{G}^{(m)}(\Omega)=\left\{\mu_{1} \ldots \mu_{m}: \mu_{k} \in \Omega\right\} .
$$

Это определение совпадает с данным ранее, если выполняется условие (1.23) и $m=2 j-1$. Пусть

$$
\widetilde{\mathbb{G}}(\Omega)=\left[\bigcup_{m=1}^{\infty} \mathbb{G}^{(m)}(\Omega)\right] \cup\left[-\bigcup_{m=1}^{\infty} \mathbb{G}^{(m)}(\Omega)\right] .
$$

Наш основной результат уточняет теорему 1.2 :

Теорема 1.6. Предположим, что вне отрезка $[-2,2]$ нет точек спектра матриць $J$ и что и - мероморбная в $\mathbb{C}$ функиия, не обращающаяся в нуль $в$ точках $z= \pm 1$. Пусть $P$ - множество полюсов функиии $u$, a $T$ - множество полюсов функиии $B$. Тогда

$$
P \subset \widetilde{\mathbb{G}}(T), \quad T \subset \widetilde{\mathbb{G}}(P) .
$$

Для того чтобы сформулировать результат в случае, когда имеются связанные состояния, мы напомним и расширим следующее понятие из [3]:

Определение. Пусть $u$ - мероморфная в $\mathbb{C}$ функция, а точка $z_{0} \in \mathbb{D}$ такова, что $u\left(z_{0}\right)=0$ (т. е. $z_{0}$ вещественна и $z_{0}+z_{0}^{-1} \in \sigma(J)$ ). Точка $z_{0}$ называется неканоническим нулем для $J$, если $1 / z_{0}$ не является полюсом функции $u$ и

$$
\lim _{z \rightarrow z_{0}}\left(z-z_{0}\right) M(z) \neq-\left(z_{0}-z_{0}^{-1}\right)\left[u^{\prime}\left(z_{0}\right) u\left(\frac{1}{z_{0}}\right)\right]^{-1} .
$$

Таким образом, если $u$ регулярна в точке $1 / z_{0}$ и выполняется условие (1.27), в котором «ұ» заменено на «=», то $z_{0}$ не является неканоническим нулем (и называется каноническим). В случае когда имеются связанные состояния или $u( \pm 1)=0$, мы доказываем следующее:

Теорема 1.7. Предположим, что и мероморфна в $\mathbb{C}$. Пусть $T$ - множество полюсов бункиии $B, P_{1}$ - множество полюсов функиии $u$, a $P_{2}=\left\{z^{-1}\right.$ : $z$ - неканонический нуль для $J\}$. Положсим $P=P_{1} \cup P_{2}$. Тогда имеют место включения (1.26).

Как и в [12], можно легко доказать утверждения, связывающие мероморфность функции $u$ в $\left\{z:|z|<R^{2 \ell-1}\right\}$ с мероморфностью функции $B$ в том же круге.

В §2 мы используем уравнения Джеронимо-Кейза для доказательства теоремы 1.5. В $\S 3$ мы пользуемся вторым отображением Сегё из ОМВП в ОМЕО 
для доказательства теоремы 1.6. В $\$ 4$ мы продолжаем анализ из [3] для того, чтобы вывести теорему 1.7 из теоремы 1.6 .

Это исследование было завершено во время моего пребывания в Еврейском университете Иерусалима в качестве приглашенного профессора Фонда леди Дэвис. Я благодарен Х. Фаркасу и Й. Ласту за гостеприимство, оказанное мне Математическим институтом этого университета.

Я с большим удовольствием посвящаю эту статью 100-летнему юбилею со дня рождения Марка Крейна. Студентом я изучал теорему Крейна-Мильмана, но только ощутив влияние М. Г. Крейна на мою собственную работу, я оценил потрясающий размах его достижений: ядерные идеалы, самосопряженные расширения (особенно для квадратичных форм), функция спектрального сдвига и его многочисленные результаты в спектральной теории ортогональных многочленов оказали на меня глубокое воздействие.

\section{§2. Уравнения Джеронимо-Кейза и $R^{-2}$-следствие}

В этом параграфе мы доказываем теорему 1.5 при помощи метода, использованного в [12] для доказательства теоремы 1.3. Там мы опирались на рекуррентное соотношение Сегё (1.13) и сопряженное к нему соотношение, т. е.

$$
\Phi_{n+1}^{*}(z)=\Phi_{n}^{*}(z)-\alpha_{n} z \Phi_{n}(z),
$$

в точках $z$ и $1 / \bar{z}$.

Здесь мы вместо этого используем уравнения Джеронимо-Кейза [5] в форме, предложенной в [3]. Положим

$$
C_{n}(z)=z^{n} P_{n}\left(z+\frac{1}{z}\right),
$$

где $P_{n}(x)=\left(\prod_{j=1}^{n} a_{j}\right) p_{n}(x)$ - нормированный ортогональный многочлен. Уравнения

$$
\begin{aligned}
& C_{n}(z)=\left(z^{2}-b_{n} z\right) C_{n-1}(z)+G_{n-1}(z), \\
& G_{n}(z)=G_{n-1}(z)+\left[\left(1-a_{n}^{2}\right) z^{2}-b_{n} z\right] C_{n-1}(z)
\end{aligned}
$$

являются ненормализованными уравнениями Джеронимо-Кейза. Вместе с начальными данными $G_{0}(z)=C_{0}(z)=1$ они определяют нормированные многочлены степени не выше $2 n$. Многочлен $C_{n}$ имеет вид $(2.2)$, и если

$$
\sum_{n=1}^{\infty}\left(\left|a_{n}^{2}-1\right|+\left|b_{n}\right|\right)<\infty
$$

то для $|z|<1$ выполняется равенство

$$
\lim _{n \rightarrow \infty} G_{n}(z)=\left(\prod_{j=1}^{\infty} a_{j}\right) u(z)
$$

(см. теорему А.3 в [3]). Мы обозначим правую часть в (2.6) через $\tilde{u}(z)$.

Соотношения (2.3), (2.4) до некоторой степени похожи на $(1.13),(2.1)$. Отличие в том, что равенство (1.14) заменено на равенство

$$
C_{n}(z)=z^{2 n} C_{n}\left(\frac{1}{z}\right),
$$


которое легко следует из (2.2). Мы будем писать $f=\widetilde{O}(g)$, где $g \rightarrow 0$, если $|f| /|g|^{1-\varepsilon} \rightarrow 0$ для всех $\varepsilon>0$.

Лемма 2.1. Если выполняется неравенство (1.21), то для $z \in \mathbb{D}$

(i) $\left|G_{n}(z)-\tilde{u}(z)\right| \leqslant \widetilde{O}\left(R^{-2 n}\right)$;

(ii) $\left|C_{n}(z)-\frac{\tilde{u}(z)}{1-z^{2}}\right| \leqslant \widetilde{O}\left(\left[\max \left(|z|, R^{-1}\right)\right]^{2 n}\right)$.

Доказательство. (i) В силу теоремы А.3 из [3]

$$
\lim _{n \rightarrow \infty} C_{n}(z)=\frac{\tilde{u}(z)}{1-z^{2}}
$$

Используя уравнения (2.4) и учитывая, что $\sup _{n}\left|C_{n}(z)\right|<\infty$, получаем

$$
\begin{aligned}
\left|G_{n}(z)-\tilde{u}(z)\right| & \leqslant \sum_{m=n}^{\infty}\left|G_{m+1}(z)-G_{m}(z)\right| \\
& \leqslant\left(\sup _{n}\left|C_{n}(z)\right|\right) \sum_{m=1}^{\infty}\left(\left|1-a_{n+m}^{2}\right|+\left|b_{n+m}\right|\right)=\widetilde{O}\left(R^{-2 n}\right),
\end{aligned}
$$

поскольку ряд сходится экспоненциально.

(ii) Из (2.3) следует, что

$$
\left|C_{n}-G_{n-1}-z^{2} C_{n-1}\right| \leqslant \sup _{n}\left|C_{n}(z)\right|\left|b_{n}\right|
$$

Итерируя, получаем

$$
\left|C_{n}-\sum_{j=0}^{n-1} G_{n-j-1} z^{2 j}\right| \leqslant|z|^{2 n}+\sup _{n}\left|C_{n}(z)\right| \sum_{j=0}^{n-1}\left|b_{n-j}\right|\left|z^{2 j}\right| \leqslant \widetilde{O}\left(\max \left(|z|, R^{-1}\right)^{2 n}\right) .
$$

В силу (2.8)

$$
\left|\sum_{j=0}^{n-1}\left(G_{n-j-1}-\tilde{u}\right) z^{2 j}\right| \leqslant \widetilde{O}\left(\max \left(|z|, R^{-1}\right)^{2 n}\right)
$$

Так как $\sum_{j} z^{2 j} u=\left(1-z^{2}\right)^{-1} u$, мы получаем (2.9).

Доказательство теоремы 1.5. Из (2.4) и (2.7) следует, что

$$
\left|G_{n+1}-G_{n}\right| \leqslant\left[\sup _{n}\left|C_{n}\left(\frac{1}{z}\right)\right|\right]|z|^{2 n+2}\left[\left|1-a_{n}^{2}\right|+\left|b_{n}\right|\right]
$$

для $|z|>1$, откуда видно, что последовательность $\left\{G_{n}\right\}_{n=0}^{\infty}$ сходится равномерно для $1<|z|<R$. В силу принципа максимума мы получаем сходимость для $|z|<R$, а значит, функция $u$ может быть аналитически продолжена в этот 
круг. В нем

$$
\begin{aligned}
\tilde{u}(z) & =1+\sum_{n=0}^{\infty}\left(G_{n+1}(z)-G_{n}(z)\right) \\
& =1+\sum_{n=0}^{\infty}\left(\left(1-a_{n+1}^{2}\right) z^{2}-b_{n+1} z\right) C_{n}(z) \\
& =\frac{\tilde{u}\left(\frac{1}{z}\right)}{1-\frac{1}{z^{2}}}(B(z)-1)+1+\sum_{n=0}^{\infty} f_{n}(z),
\end{aligned}
$$

где

$$
f_{n}(z)=\left(\left(1-a_{n+1}^{2}\right) z^{2}-b_{n+1} z\right) z^{2 n}\left(C_{n}\left(\frac{1}{z}\right)-\frac{\tilde{u}\left(\frac{1}{z}\right)}{1-\frac{1}{z^{2}}}\right) .
$$

Таким образом,

$$
\left(1-z^{2}\right) \tilde{u}(z)+\tilde{u}\left(\frac{1}{z}\right) z^{2} B(z)=\tilde{u}\left(\frac{1}{z}\right) z^{2}+\left(1-z^{2}\right)+\sum_{n=0}^{\infty}\left(1-z^{2}\right) f_{n}(z) .
$$

Каждая из функций $f_{n}$ аналитична в кольце $\{z:|z|>1\}$, так что если ряд равномерно сходится в области $\left\{z: 1<|z|<R^{2}\right\}$, то левая часть в (2.15) может быть аналитически продолжена в эту область.

В силу (2.9) для $|z|>1$

$$
\left|C_{n}\left(\frac{1}{z}\right)-\frac{\tilde{u}\left(\frac{1}{z}\right)}{1-\frac{1}{z^{2}}}\right| \leqslant \widetilde{O}\left(\max \left(\frac{1}{|z|}, R^{-1}\right)^{2 n}\right) ;
$$

поэтому

и, следовательно,

$$
\left|z^{2 n}\left[C_{n}\left(\frac{1}{z}\right)-\frac{\tilde{u}\left(\frac{1}{z}\right)}{1-\frac{1}{z^{2}}}\right]\right| \leqslant \widetilde{O}\left(\max \left(1,|z| R^{-1}\right)^{2 n}\right)
$$

$$
\left|f_{n}(z)\right| \leqslant \widetilde{O}\left(R^{-2 n}\right) \widetilde{O}\left(\max \left(1,|z| R^{-1}\right)^{2 n}\right) .
$$

Для $1<|z|<R$ величина в правой части есть $\widetilde{O}\left(R^{-2 n}\right)$, а значит, левые части суммируемы. Для $R \leqslant|z|<R^{2}$ правая часть есть $\widetilde{O}\left(\left(|z| R^{-2}\right)^{2 n}\right)$ и левые части также суммируемы.

В случае когда $u\left( \pm R^{-1}\right) \neq 0$, из $(1.22)$ вытекает, что так как $B$ имеет особенности на окружности радиуса $R$, то $u$ также должна иметь особенности на этой окружности. Однако если $u\left(R^{-1}\right)=0$ и/или $u\left(-R^{-1}\right)=0$, то этот нуль может компенсировать полюс функции $B$, и $u$ может иметь большую область аналитичности, чем $B$. Как показано в [3], именно это происходит в случае неканонических весов.

\section{§3. Второе отображение Сегё и функции Йоста без связанных состояний}

В [13], [14] Сегё ввел два отображения вероятностных мер на $\partial \mathbb{D}$, инвариантных относительно комплексного сопряжения, в вероятностные меры на $[-2,2]$; мы обозначаем их через $\mathrm{Sz}_{1}$ и $\mathrm{Sz}_{2}$. Оба эти отображения инъективны, но только $\mathrm{Sz}_{1}$ сюръективно. Поэтому именно $\mathrm{Sz}_{1}$ чаще всего используется и изучается (см. 
[11, разд. 13.1]). Однако мы увидим, что отображение $\mathrm{Sz}_{2}$ также чрезвычайно полезно, особенно для изучения функций Йоста, аналитических в окрестности круга $\overline{\mathbb{D}}$ и не имеющих нулей в $\overline{\mathbb{D}}$ (случай, когда $J$ не имеет ни резонансов в \pm 2 , ни связанных состояний).

Для абсолютно непрерывных мер эти отображения задаются следующими соотношениями:

$$
d \mu=w(\theta) \frac{d \theta}{2 \pi}, \quad \mathrm{Sz}_{1}(d \mu)=f_{1}(x) d x, \quad \mathrm{Sz}_{2}(d \mu)=f_{2}(x) d x,
$$

где $w(\theta)=w(-\theta)$ и (см. формулы (13.1.6) и (13.2.22) в [11])

$$
\begin{aligned}
& f_{1}(x)=\pi^{-1}\left(4-x^{2}\right)^{-1 / 2} w(\arccos (x / 2)), \\
& f_{2}(x)=\pi^{-1} c^{2}\left(4-x^{2}\right)^{1 / 2} w(\arccos (x / 2)) ;
\end{aligned}
$$

здесь

$$
c=\left[2\left(1-\left|\alpha_{0}\right|^{2}\right)\left(1-\alpha_{1}\right)\right]^{-1 / 2} .
$$

Принимая во внимание, что $\mathrm{Sz}_{1}$ - биекция между симметричными мерами на $\partial \mathbb{D}$ и произвольными мерами на $[-2,2]$, мы получаем, что

$$
d \gamma \in \operatorname{ran}\left(\mathrm{Sz}_{2}\right) \Longleftrightarrow \int_{-2}^{2}\left(4-x^{2}\right)^{-1} d \gamma(x)<\infty .
$$

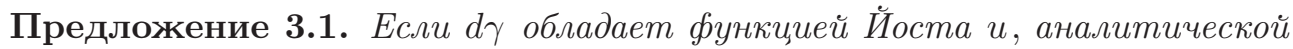
в окрестности круга $\overline{\mathbb{D}}$ и не обращающейся в нуль в $\overline{\mathbb{D}}, m о d \gamma \in \operatorname{ran}\left(\mathrm{Sz}_{2}\right)$.

Доказательство. Так как $d(2 \cos \theta)=-2 \sin \theta d \theta$ и $\left(4-4 \cos ^{2} \theta\right)=4 \sin ^{2} \theta$, в силу (1.4) правая часть в (3.5) эквивалентна соотношению

$$
\frac{1}{\pi} \int_{0}^{\pi} \frac{d \theta}{\left|u\left(e^{i \theta}\right)\right|^{2}}=\int_{0}^{\pi}\left(\sin ^{2} \theta\right)^{-1} f(2 \cos \theta) \sin \theta d \theta=2 \int_{-2}^{2}\left(4-x^{2}\right)^{-1} f(x) d x<\infty,
$$

которое верно, если функция $|u|$ отделена от нуля.

Для наших целей важна

Теорема 3.2. Предположим, что $d \mu$ является нетривиальной вероятностной мерой на $\partial \mathbb{D}$, удовлетворяющей условию Сегё, с коэффициентами Верблунского $\left\{\alpha_{n}\right\}_{n=0}^{\infty}$ и функиией Сегё $D\left(z_{0}\right)$. Пусть мере $d \gamma=\mathrm{Sz}_{2}(d \mu)$ соответствуют функиия Йоста и и параметры Якоби $\left\{a_{n}, b_{n}\right\}_{n=1}^{\infty}$. Тогда

$$
\begin{aligned}
b_{n+1} & =\alpha_{2 n}-\alpha_{2 n+2}-\alpha_{2 n+1}\left(\alpha_{2 n}+\alpha_{2 n+2}\right), \\
a_{n+1}^{2}-1 & =\alpha_{2 n+1}-\alpha_{2 n+3}-\alpha_{2 n+2}^{2}\left(1-\alpha_{2 n+3}\right)\left(1+\alpha_{2 n+1}\right)-\alpha_{2 n+3} \alpha_{2 n+1}, \\
u(z) & =\left(1-\left|\alpha_{0}\right|^{2}\right)\left(1-\alpha_{1}\right) D(z)^{-1} .
\end{aligned}
$$

Замечание. Формулы вида (3.6), (3.7) для $\mathrm{Sz}_{1}$ восходят к Геронимусу [6], [7]. Для $\mathrm{Sz}_{2}$ наиболее ранней из известных мне ссылок является [1]; см. также [8].

Доказательство. Уравнения (3.6), (3.7) совпадают с уравнениями (13.2.20), (13.2.21) в [11]. Для доказательства равенства (3.8) заметим, что в силу (1.4) и

$$
\left|u\left(e^{i \theta}\right)\right|^{-2}=\pi f_{2}(2 \cos \theta)(\sin \theta)^{-1}=2 c^{2} w=2 c^{2}|D|^{2} .
$$


Следовательно, если $z=e^{i \theta}$, то модули правой и левой частей в (3.8) равны. Поскольку обе части аналитичны, не имеют нулей в $\mathbb{D}$ и положительны в $z=0$, равенство (3.8) выполняется при всех $z$.

Тождества (3.6), (3.7), прежде всего, дают второе доказательство теоремы 1.5 в случае, когда $u$ не имеет нулей в $\overline{\mathbb{D}}$, и, что более важно, показывают, что в общем случае граница $R^{2}$ оптимальна. Докажем сначала

Предложение 3.3. Имеет место равенство

$$
B(z)=\alpha_{0} z^{-1}+\alpha_{1}+1+(S(z)-1)\left(1-z^{-2}\right)+Q(z),
$$

где $Q$ аналитична в $\left\{z:|z|<R^{2}\right\}$, если

$$
\limsup _{n \rightarrow \infty}\left|\alpha_{n}\right|^{1 / n}=R^{-1} .
$$

Доказательство. Используя (1.9), (1.15) и (3.6), (3.7), мы получаем (3.9), где

$$
\begin{aligned}
Q(z)=\sum_{n=0}^{\infty} \alpha_{2 n+1} & \left(\alpha_{2 n}+\alpha_{2 n+2}\right) z^{2 n+1} \\
& +\left\{\alpha_{2 n+2}^{2}\left(1-\alpha_{2 n+3}\right)\left(1+\alpha_{2 n+1}\right)+\alpha_{2 n+3} \alpha_{2 n+1}\right\} z^{2 n+2} .
\end{aligned}
$$

В силу условия (3.10) $Q(z)$ аналитична в $\left\{z:|z|<R^{2}\right\}$.

Второе доказательство теоремы 1.5 в случае, когда $u$ не имеет нулей в $\overline{\mathbb{D}}$. Как мы вскоре увидим (см. лемму 3.5$)$, из включений (1.26) следует равенство (3.10). Используя теорему 1.3 и равенство (3.8), мы заключаем, что функция

$$
\left(z^{2}-1\right)[u(z)-\overline{u(1 / \bar{z})} S(z)]
$$

аналитична в $\left\{z: R^{-1}<|z|<R^{3}\right\}$. В силу предложения 3.3 функция

$$
z^{2} B(z)-\left(z^{2}-1\right) S(z)
$$

аналитична в $\left\{z:|z|<R^{2}\right\}$, так что установленная выше аналитичность функции (3.12) дает аналитичность функции (1.22) в $\left\{z: R^{-1}<|z|<R^{2}\right\}$.

Пример 3.4. Пусть $\alpha_{2 n} \equiv 0$ (что верно в том и только том случае, когда $\left.b_{n} \equiv 0\right)$ и $\alpha_{2 n+1}=R^{-(2 n+1)}$. Тогда в силу (3.10) функция

$$
Q(z)=\sum_{n=0}^{\infty} z^{2 n+2} R^{-4 n-4}=z^{2} R^{-4}\left(1-z^{2} R^{-4}\right)^{-1}
$$

имеет полюсы в $z= \pm R^{2}$. Это показывает, что функция (1.22) может не быть аналитичной ни в каком более широком кольце, чем $\left\{z: R^{-1}<|z|<R^{2}\right\}$. При помощи аналогичного анализа получаем, что если $B$ мероморфна в $\{z:|z|<$ $\left.R^{1+\varepsilon}\right\}$, то, вообще говоря, функция (1.22) будет иметь особенности на окружности радиуса $R^{2}$. Замена $R^{3}$ на $R^{2}$, произошедшая при переходе от теоремы 1.3 к теореме 1.5, обусловлена квадратичными слагаемыми в (3.6), (3.7).

Далее нам будет нужна (уже использованная выше)

Лемма 3.5. Пусть $\left\{\alpha_{n}\right\}_{n=0}^{\infty} u\left\{a_{n}, b_{n}\right\}_{n=1}^{\infty}$ связаны посредством формул (3.6), (3.7) u

$$
\limsup _{n \rightarrow \infty}\left|\alpha_{n}\right|^{1 / n} \equiv R_{1}^{-1}<1, \quad \limsup _{n \rightarrow \infty}\left(\left|a_{n}^{2}-1\right|+\left|b_{n}\right|\right)^{1 / 2 n} \equiv R_{2}^{-1}<1 .
$$


Тогда $R_{1}=R_{2}$. Кроме того, $\left\{\alpha_{n}\right\}_{n=0}^{\infty}$ обладает полным асимптотическим разложением тогда и только тогда, когда $\left\{a_{n}, b_{n}\right\}_{n=1}^{\infty}$ обладают таким разложением, и если $T$ - множество степеней, входящих в разложение для $\left\{a_{n}, b_{n}\right\}_{n=1}^{\infty}$ (т.е. $T$ - множество полюсов функции $\left.B\right)$, а $\widetilde{T}-$ аналогичное множество для $\left\{\alpha_{n}\right\}_{n=0}^{\infty}$ (т.е. $T$ - множество полюсов функиии $\left.S\right)$, то

$$
T \subset \widetilde{\mathbb{G}}(\widetilde{T}), \quad \widetilde{T} \subset \widetilde{\mathbb{G}}(T) .
$$

Замечание. Решения уравнений для $\mathrm{Sz}_{1}$, аналогичных уравнениям (3.6), (3.7), таковы, что $\left\{a_{n}, b_{n}\right\}_{n=1}^{\infty}$ быстро убывают, в то время как $\alpha_{2 n+1} \sim n^{-1}$ при $n \rightarrow \infty$. (В самом деле, для $\mathrm{Sz}_{1}$, но не для $\mathrm{Sz}_{2}$ это происходит с $J_{0}$; см. пример 13.1.3 в [11].) Фактически из результатов этой работы и работы [12] вытекает, что $R_{1}^{-1}<1$ в том и только том случае, когда $R_{2}^{-1}<1$.

Доказательство. Из (3.6) следует, что если $R_{1}^{-1}, R_{2}^{-1}<1$, то $R_{2}=R_{1}$, поскольку неглавные слагаемые экспоненциально малы. Кроме того, если $\alpha_{n}$ обладает полным асимптотическим разложением, то $b_{n+1}$ и $a_{n+1}^{2}-1$ по отдельности обладают асимптотическими разложениями по $\mu_{k}^{-2 n}$, где $\mu_{k} \in \bigcup_{j=1}^{\infty} \mathbb{G}^{(j)}(\widetilde{T})$. Так как

$$
\begin{aligned}
c_{1} \mu_{k}^{-2 n} & =\frac{1}{2}\left(c_{1}+c_{2}\right) \mu_{k}^{-2 n}+\frac{1}{2}\left(c_{1}-c_{2}\right)\left(-\mu_{k}\right)^{-2 n}, \\
c_{2} \mu_{k}^{-2 n-1} & =\frac{1}{2}\left(c_{1}+c_{2}\right) \mu_{k}^{-2 n-1}+\frac{1}{2}\left(c_{1}-c_{2}\right)\left(-\mu_{k}\right)^{-2 n-1},
\end{aligned}
$$

мы можем объединить их в одно разложение, используя $-\mu_{k}$ наравне с $\mu_{k}$.

Чтобы доказать обратное, отметим, что экспоненциальное убывание последовательности $\alpha_{n}$ приводит к равенствам

$$
\alpha_{2 n}=\sum_{m=0}^{\infty} b_{n+m+1}+O\left(R^{-2 n}\right)
$$

и аналогичным равенствам для $\alpha_{2 n+1}$ и $\sum_{m=0}^{\infty}\left(a_{n+m+1}^{2}-1\right)$. Подставляя в $(3.6)$ и суммируя, мы получаем явные выражения для $\alpha_{2 n}$ и $\alpha_{2 n+1}$ в виде сумм произведений четырех или меньшего числа параметров $b$ и $1-a^{2}$ с точностью до $O\left(R^{-3 n}\right)$. Итерируя, выводим отсюда явные представления для $\alpha$ в виде «многочленов» от $b$ и $1-a^{2}$ степени $k$ с точностью до $O\left(R^{-(k+2) n}\right)$. Это показывает, что если $a$ и $b$ обладают асимптотическими разложениями с точностью до $O\left(R^{-(k+1) n}\right)$, то это же верно для $\alpha_{2 n}$ и $\alpha_{2 n+1}$, причем соответствующие $\mu$ (основания степеней) лежат в $\bigcup_{j=1}^{\infty} \mathbb{G}^{(j)}(T)$. При помощи формул, аналогичных (3.16), (3.17), мы можем объединить эти два разложения в одно, используя $-\mu$ наравне с $\mu$. Таким образом, $\widetilde{T} \subset \widetilde{G}(T)$.

Доказательство теорем 1.6 и 1.2 в случае, когда $u$ не имеет нулей в $\overline{\mathbb{D}}$. Если $u$ не имеет нулей в $\overline{\mathbb{D}}$, то $\gamma \in \operatorname{ran}\left(\mathrm{Sz}_{2}\right)$ и мы можем определить $S$, $\alpha_{n}$ и т. д. В силу (3.8), если $u$ мероморфна во всей комплексной плоскости, то такова же и $D^{-1}$. Значит, по теореме 1.4 функция $S$ мероморфна в $\mathbb{C}$, и если $\widetilde{T}-$ множество ее полюсов, то

$$
\widetilde{T} \subset \mathbb{G}(P) .
$$

В силу леммы 3.5 функция $B$ мероморфна и

$$
T \subset \widetilde{\mathbb{G}}(\widetilde{T}) \subset \widetilde{\mathbb{G}}(\mathbb{G}(P))=\widetilde{\mathbb{G}}(P) .
$$


Обратно, если $B$ мероморфна во всей комплексной плоскости, то по лемме 3.5 такова же и $S$, и если $\widetilde{T}-$ множество полюсов функции $S$, то

$$
\widetilde{T} \subset \widetilde{\mathbb{G}}(T) .
$$

По теореме 1.4 функция $D^{-1}$, а значит, и $u$ мероморфны в $\mathbb{C}$ и

$$
P \subset \mathbb{G}(\widetilde{T}) \subset \mathbb{G}(\widetilde{\mathbb{G}}(T))=\widetilde{\mathbb{G}}(T) .
$$

\section{§4. Сдвиг коэффициентов и функции Йоста со связанными состояниями}

Как и в [3], мы переходим от случая, когда отсутствуют связанные состояния, к общему случаю (т. е. от теоремы 1.6 к теореме 1.7) при помощи сдвига коэффициентов («процедуры раздевания»), т. е. при помощи перехода от $J$ к матрице Якоби $J^{(m)}$ с параметрами $\left\{a_{n+m}, b_{n+m}\right\}_{n=1}^{\infty}$. Теорема 3.1 из [3] утверждает, что если функция Йоста, соответствующая матрице $J$, аналитична в окрестности круга $\overline{\mathbb{D}}$, то найдется такое $k$, что $\sigma\left(J^{(k)}\right)=[-2,2]$. Используя небольшое дополнительное рассуждение, мы также можем считать, что для новой матрицы Якоби $u^{(k)}( \pm 1) \neq 0$ (ибо если $\sigma\left(J^{(k-1)}\right)=[-2,2]$ и $u^{(k-1)}$ имеет корень в \pm 1 , то $M^{(k-1)}(z)$ имеет там же полюс, а значит, $u^{(k)}=u^{(k-1)} M^{(k-1)}$ не обращается в нуль). Таким образом, мы утверждаем, что все, что нам требуется (и что мы докажем ниже), - это следующий факт:

Теорема 4.1. Если множества $P=P_{1} \cup P_{2}$ такие, как в теореме 1.7, то

$$
P(J)=P\left(J^{(1)}\right),
$$

где мы указываем зависимость от $J$ явно.

Вывод теорем 1.1 и 1.7 из теорем 4.1 и 1.6. Теорема 1.7 влечет за собой теорему 1.1. Используя равенство (4.1) и индукцию, видим, что $P(J)=P\left(J^{(k)}\right)$, где $k$ выбрано таким образом (см. выше), чтобы была применима теорема 1.6. Теперь включения (1.26) для $J$ следуют из включений $(1.26)$ для $J^{(k)}$.

Как и в [3], мы будем использовать $M$-функцию, ее связь с $u$ и рекуррентные соотношения. Положим (в соответствии с (1.5))

$$
M^{(k)}(z)=\left\langle\delta_{1},\left(z+z^{-1}-J^{(k)}\right)^{-1} \delta_{1}\right\rangle
$$

для $z \in \mathbb{D} \backslash\left\{w: w+w^{-1} \in \sigma\left(J^{(k)}\right)\right\}$. Функция $M^{(k)}$ имеет полюсы в таких точках $w$ из $\mathbb{D}$, что $w+w^{-1} \in \sigma\left(J^{(k)}\right)$, а $u^{(k)}$ имеет в этих же точках нули. Справедливы (исходно для $z \in \mathbb{D}$ ) рекуррентные соотношения (см. (2.4), (2.5) в [3])

$$
\begin{aligned}
u^{(k+1)}(z) & =a_{k+1} z^{-1} u^{(k)}(z) M^{(k)}(z), \\
M^{(k)}(z)^{-1} & =z+z^{-1}-b_{k+1}-a_{k+1}^{2} M^{(k+1)}(z) .
\end{aligned}
$$

Кроме того, если $z=e^{i \theta}, \theta \in[0, \pi]$, то из (1.4) и соотношения $\pi f(2 \cos \theta)=$ $\operatorname{Im} M\left(e^{i \theta}\right)$ вытекает, что

$$
[M(z)-\bar{M}(1 / \bar{z})] \overline{u(1 / \bar{z})} u(z)=z-z^{-1} .
$$

Тождество (4.5) может быть использовано для мероморфного продолжения функции $M$ из $\mathbb{D}$ в $\mathbb{C}$, если $u$ мероморфна во всей комплексной плоскости. 
После того как мы построили это продолжение, соотношения (4.3), (4.4) распространяются на все $z \in \mathbb{C}$ (как равенства мерофорфных функций, с учетом возможных сокращений полюсов и нулей). Эти соотношения также гарантируют мероморфность функции $u^{(1)}$ в $\mathbb{C}$, если $u$ мероморфна в $\mathbb{C}$.

Начнем с эквивалентного определения множества $P_{2}$ :

Предложение 4.2. Точка $z_{0} \in \mathbb{C} \backslash \overline{\mathbb{D}}$ лежит в $P_{2}$ тогда и толъко тогда, когда

(i) $z_{0}$ не является полюсом функиии $и$;

(ii) обе точки $z_{0}$ и $z_{0}^{-1}$ являются полюсами функиии $M$.

Замечание. Все полюсы функции $M$, лежащие в $\mathbb{D}$, вещественны, а значит, из (ii) вытекает вещественность $z_{0}$.

Доказательство. По определению $z_{0} \in P_{2}$, если $u\left(z_{0}^{-1}\right)=0, z_{0}$ не является полюсом функции $u$ и выполняется (1.27). Так как $z_{0}^{-1} \in \mathbb{D}$, то $u\left(z_{0}^{-1}\right)=0 \Longleftrightarrow z_{0}+z_{0}^{-1} \in \sigma(J) \Longleftrightarrow z_{0}^{-1}$ является полюсом функции $M(z)$.

Как показано в [3], в силу (4.5), если $u\left(z_{0}\right)=0$, то $z_{0}$ является полюсом функции $M$ порядка два или более и, конечно, соотношение (1.27) выполняется в $z_{0}^{-1}$, поскольку его левая часть бесконечна. Если $u\left(z_{0}\right) \neq 0$, то $(1.27)$ в точности (с учетом равенства (4.5)) означает, что $M(z)$ имеет полюс в $z_{0}$.

Мы уже отмечали случай, когда $z_{0}$ является полюсом функции $u$, в то время как $z_{0}^{-1}$ является ее нулем. Мы должны исследовать его отдельно:

Предложение 4.3. Если $z_{0} \in \mathbb{C} \backslash \mathbb{D}$ является полюсом функиии и, а $z_{0}^{-1}-$ ее нулем, то $z_{0}$ является полюсом функиии $u^{(1)}$.

Доказательство. Рассмотрим равенство (4.5) около точки $z=z_{0}$. Так как все нули функции $u$ в $\mathbb{D}$ простые, то $u(z) \overline{u(1 / \bar{z})}$ имеет в $z_{0}$ либо полюс, либо конечный ненулевой предел. Тогда (4.5) гарантирует регулярность функции $M(z)-\overline{M(1 / \bar{z})}$ в $z_{0}$ (возможно, эта функция даже имеет в $z_{0}$ нуль). Так как $\overline{M(1 / \bar{z})}$ имеет полюс в $z_{0}, M(z)$ также должна иметь в $z_{0}$ полюс. Следовательно, $u^{(1)}=a_{1} z^{-1} u M$ имеет полюс (как минимум второго порядка) в $z_{0}$.

Предложение 4.4. Если $z_{0} \in P_{2}(J)$, то $z_{0} \in P_{1}\left(J^{(1)}\right)$.

Замечания. 1. Этот результат для точки $z_{0}$ внутри круга аналитичности функции $u$ содержится в [3]. Мы приводим здесь практически то же самое доказательство.

2. Утверждение, что $z_{0} \in P_{2}(J)$, в сущности, означает, что «резонансная собственная функция» обращается в нуль, и, таким образом, мы утверждаем, что такие собственные функции не могут иметь двух последовательных нулей и удовлетворять при этом разностному уравнению второго порядка.

Доказательство. Предположим сначала, что $u\left(z_{0}\right) \neq 0$. Согласно предложению $4.2, M$ имеет полюс в $z_{0}$; поэтому $u^{(1)}=a_{1} z^{-1} u M$ также имеет полюс B $z_{0}$.

Если $u$ имеет нуль $k$-го $(k \geqslant 1)$ порядка в $z_{0}$, то $\overline{u(1 / \bar{z})} u(z)$ имеет в этой точке нуль $(k+1)$-го порядка, а значит, в силу $(4.5) M(z)-\overline{M(1 / \bar{z})}$ имеет полюс $(k+1)$-го порядка в $z_{0}$. Поскольку функция $M$ имеет простые полюсы в 
точках области $\mathbb{D}$ типа $1 / z_{0}$, она обязана иметь полюс $(k+1)$-го порядка в $z_{0}$. Таким образом, $u^{(1)}=a_{1} z^{-1} u M$ имеет полюс в $z_{0}$.

Предложение 4.5. Если $z_{0} \in P_{1}(J) u z_{0} \notin P_{1}\left(J^{(1)}\right)$, mo $z_{0} \in P_{2}\left(J^{(1)}\right)$.

Доказательство. Из (4.4) следует, что полюсы функции $M^{(1)}(z)$ являются в точности нулями функции $M(z)$. Следовательно, в силу предложения 4.2 нам нужно только доказать, что

$$
\text { если } z_{0} \in P_{1}(J) \text { и } z_{0} \notin P_{1}\left(J^{(1)}\right) \text {, то } M\left(z_{0}\right)=M\left(1 / z_{0}\right)=0 .
$$

Так как $u$ имеет полюс в $z_{0}$, а $u^{(1)}=a_{1} z^{-1} u M$ не имеет, то $M\left(z_{0}\right)=0$. В силу предложения 4.3 , если $z_{0} \notin P_{1}\left(J^{(1)}\right)$, то $u(1 / \bar{z}) \neq 0$. Значит, $u(z) \overline{u(1 / \bar{z})}$ имеет полюс в $z_{0}$. Тогда из тождества (4.5) вытекает, что $M(z)-\left.\overline{M(1 / \bar{z})}\right|_{z=z_{0}}=0$. Поскольку $M\left(z_{0}\right)=0$, мы получаем $M\left(1 / \bar{z}_{0}\right)=0$, что и доказывает утверждение (4.6).

Нам также нужно доказать некоторые утверждения в обратную сторону, т. е. исходящие из того, что $z_{0} \in P\left(J^{(1)}\right)$.

Предложение 4.6. Если $z_{0} \in P_{2}\left(J^{(1)}\right)$, mo $z_{0} \in P_{1}(J)$.

Доказательство. Согласно предложению 4.2, $z_{0}$ и $z_{0}^{-1}$ являются полюсами функции $M^{(1)}$, а значит, в силу (4.4) - нулями функции $M$. Как и в доказательстве предложения 4.4, если $u^{(1)}$ имеет в $z_{0}$ нуль $k$-го порядка (включая случай $k=0$, т. е. $\left.u^{(1)}\left(z_{0}\right) \neq 0\right)$, то $M^{(1)}(z)$ имеет в этой же точке полюс $(k+1)$ ого порядка, а значит, $M(z)$ имеет нуль $(k+1)$-го порядка. Это согласуется с равенством $u^{(1)}=a_{1} z^{-1} u M$, только если $u$ имеет полюс в $z_{0}$.

Предложение 4.7. Если $z_{0} \in P_{1}\left(J^{(1)}\right)$ u $z_{0} \notin P_{1}(J)$, mo $z_{0} \in P_{2}(J)$.

Доказательство. По предположению $z_{0}$ не является полюсом функции $u$, и, значит, выполняется первое условие предложения 4.2. Таким образом, нам требуется только показать, что $M(z)$ имеет полюсы в $z_{0}$ и $z_{0}^{-1}$. Предположим, что $u$ имеет нуль $k$-го порядка в $z_{0}$ (включая случай $k=0$, т. е. когда $\left.u\left(z_{0}\right) \neq 0\right)$. Используя (4.3) и тот факт, что $z_{0}$ является полюсом функции $u^{(1)}$, мы видим, что $z_{0}$ является полюсом $(k+1)$-го порядка функции $M(z)$ и, в частности, ее полюсом (так как $k+1 \geqslant 1)$.

Если $k \geqslant 1$, то единственная возможность, согласующаяся с равенством (4.5), - это то, что $\overline{u(1 / \bar{z})}$ имеет нуль в $z_{0}$, поскольку возможный полюс функции $M$ в $z_{0}^{-1}$ может быть только простым и не может компенсировать полюс $(k+1)$-го порядка в $z_{0}$. Значит, $\overline{u\left(1 / \bar{z}_{0}\right)}=0$, а тогда точка $z_{0}^{-1}$ лежит на вещественной оси и является полюсом функции $M(z)$. Таким образом, второе условие предложения 4.2 также выполняется и $z_{0} \in P_{2}(J)$.

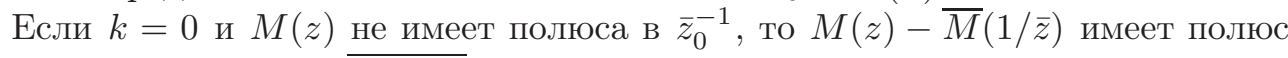
в $z_{0}$, в то время как $\overline{u\left(1 / \bar{z}_{0}\right)} \neq 0 \neq u\left(z_{0}\right)$ (поскольку $k=0$ и $1 / \bar{z}_{0}$ не есть полюс), что противоречит (4.5). Следовательно, $M$ должна иметь полюс в $\bar{z}_{0}^{-1}$, и $z_{0} \in P_{2}(J)$ в силу предложения 4.2 .

Доказательство теоремы 4.1. Если $z_{0} \in P\left(J^{(1)}\right)$, то либо $z_{0} \in P_{2}\left(J^{(1)}\right)$, а значит, $z_{0} \in P_{1}(J)$ (в силу предложения 4.6), либо $z_{0} \in P_{1}\left(J^{(1)}\right)$, а значит, $z_{0} \in P_{1}(J) \cup P_{2}(J)$ (в силу предложения 4.7). Следовательно, $P\left(J^{(1)}\right) \subset P(J)$. 
Если $z_{0} \in P(J)$, то либо $z_{0} \in P_{2}(J)$, а значит, $z_{0} \in P_{1}\left(J^{(1)}\right)$ (в силу предложения 4.4), либо $z_{0} \in P_{1}(J)$, а значит, $z_{0} \in P_{1}\left(J^{(1)}\right) \cup P_{2}\left(J^{(1)}\right)$ (в силу предложения 4.5). Поэтому $P(J) \subset P\left(J^{(1)}\right)$.

\section{ЛитератУра}

[1] E. Berriochoa, A. Cachafeiro, J. García-Amor, Generalizations of the Szegö transformation interval-unit circle, preprint.

[2] D. Damanik, B. Simon, Jost functions and Jost solutions for Jacobi matrices, I. A necessary and sufficient condition for Szegö asymptotics, Invent. Math., 165 (2006), 1-50.

[3] D. Damanik, B. Simon, Jost functions and Jost solutions for Jacobi matrices, II. Decay and analyticity, Internat. Math. Res. Notices, 2006, Art. ID 19396.

[4] P. Deift, J. Östensson, A Riemann-Hilbert approach to some theorems on Toeplitz operators and orthogonal polynomials, J. Approx. Theory, 139 (2006), 144-171.

[5] J. S. Geronimo, K. M. Case, Scattering theory and polynomials orthogonal on the unit circle, J. Math. Phys., 20 (1979), 299-310.

[6] Ya. L. Geronimus, On the trigonometric moment problem, Ann. of Math. (2), 47 (1946), 742-761.

[7] Я. Л. Геронимус, Полиномь, ортогональнье на круге, и их приложения, Зап. научно-исслед. инст. мат. мех. Харьков. матем. общ. (4), 19 (1948), 35-120.

[8] R. Killip, I. Nenciu, Matrix models for circular ensembles, Internat. Math. Res. Notices, 50 (2004), 2665-2701.

[9] R. Killip, B. Simon, Sum rules for Jacobi matrices and their applications to spectral theory, Ann. of Math. (2), 158 (2003), 253-321.

[10] B. Simon, Orthogonal Polynomials on the Unit Circle, Part 1: Classical Theory, part 1, Amer. Math. Soc. Colloq. Publ., vol. 54, Amer. Math. Soc., Providence, RI, 2005.

[11] B. Simon, Orthogonal Polynomials on the Unit Circle, Part 2: Spectral Theory, part 2, Amer. Math. Soc. Colloq. Publ., vol. 54, Amer. Math. Soc., Providence, RI, 2005.

[12] B. Simon, Meromorphic Szegö functions and asymptotic series for Verblunsky coefficients, Acta Math., 195 (2005), 267-285.

[13] G. Szegö, Über den asymptotischen Ausdruck von Polynomen, die durch eine Orthogonalitätseigenschaft definiert sind, Math. Ann., 86 (1922), 114-139.

[14] G. Szegö, Orthogonal Polynomials, Amer. Math. Soc. Colloq. Publ., vol. 23, Amer. Math. Soc., Providence, RI, 1939; 3rd edition, 1967.

California Institute of Technology, Pasadena e-mail: bsimon@caltech.edu 